\title{
A Major QTL Confers Rapid Internode Elongation in Response to Water Rise in Deepwater Rice
}

\author{
Yoko Hattori ${ }^{1,2)}$, Kotaro Miura ${ }^{1,2)}$, Kenji Asano ${ }^{1,2)}$, Eiji Yamamoto ${ }^{1)}$, Hitoshi Mori' ${ }^{3)}$, Hidemi Kitano ${ }^{1)}$, \\ Makoto Matsuoka ${ }^{1)}$ and Motoyuki Ashikari*1) \\ 1) Bioscience and Biotechnology Center, Nagoya University, Furo, Chikusa, Nagoya, Aichi 464-8601, Japan \\ 2) Japan Society for the Promotion of Science, 8 Ichibancho, Chiyoda, Tokyo 102-8472, Japan \\ 3) Graduate School of Bioagricultural Sciences, Nagoya University, Furo, Chikusa, Nagoya, Aichi 464-8601, Japan
}

To avoid drowning under flooded conditions, deepwater rice responds to rising water level by rapid internode elongation. Quantitative trait locus (QTL) analysis, using a deepwater rice cultivar (Oryza sativa) and a wild rice species (O. rufipogon) with deepwater characteristics, revealed the presence of major QTLs (qTIL12, $q N E I 12$ and $q L E I 12)$ in a common chromosomal region that regulates internode elongation. Genetic analysis revealed that a QTL inherited in a dominant manner, was located on the long arm of chromosome 12. A nearly isogenic line (NIL), produced by backcross introduction of a chromosome fragment carrying this major QTL into non-deepwater rice, exhibited a dramatic internode elongation in response to water rise. This indicates that the difference between deepwater rice and non-deepwater rice is associated with the presence of the QTL, and that this sequence is sufficient to confer deepwater characteristics. Although deepwater rice and non-deepwater rice share a common machinery for internode elongation; non-deepwater rice cannot activate the machinery in response to flooding, unlike deepwater rice which harbors this major QTL.

Key Words: rice, deepwater, QTL.

\section{Introduction}

Deepwater rice, or floating rice, is mainly cultivated in the lowland areas of South and Southeast Asia where flooding occurs during the rainy season. Under flooded conditions, deepwater rice plants develop extremely long stems due to rapid internode elongation and on increase in the number of internodes. Although deepwater rice does not display significant internode elongation in air during the vegetative stage; under flooded conditions, rising water level induces rapid internode elongation that allows the plant to keep its leaves above water for respiration. Remarkable internode growth occurs under flooded conditions, with daily increases in plant height of 20 to $25 \mathrm{~cm}$. Ultimately, the plants can reach a total maximum height of up to $7 \mathrm{~m}$, in contrast to only about $1-1.5 \mathrm{~m}$ when they are air-grown (Vergara et al. 1976, Catling 1992). Apart from this unusual growth characteristic, however, the general morphology of deepwater rice appears to be similar to that of ordinary paddy rice (Hamamura 1993).

Morphological and physiological analyses of deepwater rice have shown that phytohormones such as ethylene, abscisic acid and gibberellin are involved in rapid internode elongation (Kende et al. 1998). In particular, ethylene has

Communicated by Q. Qian

Received April 27, 2007. Accepted July 19, 2007.

*Corresponding author (e-mail: ashi@agr.nagoya-u.ac.jp) been identified as an initial factor for internode elongation. Métraux and Kende (1983) reported that the level of intralacunar ethylene in plants increased by submergence, and the application of ethylene inhibitors suppressed internode growth. Raskin and Kende (1984) reported that at low $\mathrm{O}_{2}$ concentrations, $1 \mu \mathrm{L} / \mathrm{L}$ ethylene stimulated internode elongation. Molecular biological approaches have revealed that the expression of several deepwater rice genes is regulated by the rise of the water level (Cho and Kende 1997, van der Knaap et al. 1997, 1999, 2000, Zarembinski and Theologis 1997, Vriezen et al. 1999, Lee and Kende 2001, 2002, Zhou et al. 2001). However, the exact trigger(s) for rapid internode elongation is unknown. While physiological and molecular biological approaches have been used to identify the gene(s) involved in internode elongation, few studies have relied on a genetic approach. Ramiah and Ramaswami (1940) suggested that the elongation process is controlled by duplicate genes, designated as efl and ef2, while Hamamura and Kupkanchankul (1979) reported that the ability of rice to float was a partially dominant character. On the other hand, Tripathi and Balakrishna Rao (1985) reported that early nodal differentiation, a characteristic of deepwater rice, is controlled by a single dominant gene, whereas Suge (1987) reported that internodal elongation in deepwater rice can be explained by the presence of complementary genes. Finally, Eiguchi et al. (1993) reported that a single recessive gene, $d w 3$, was associated with internode elongation in deepwater rice. These results are not only inconsistent, but also none of 
the genes have been mapped. Furthermore, since the gene(s) that triggers rapid internode elongation in deepwater rice has not yet been identified; the mechanism remains unclear.

To understand the precise molecular mechanism of rapid internode elongation in deepwater rice in response to water rise, the gene(s) that initiates the process must be identified and cloned. Many agronomic traits, including plant height, displayed broad phenotypic variations among the progenies of a single cross (Ashikari et al. 2005, Ashikari and Matsuoka 2006). Such complex traits are generally controlled by sets of genes known as quantitative trait loci (QTLs), which are derived from natural variation (Yano and Sasaki 1997, Yano 2001). QTL analysis enables the identification and cloning of genes that control complex traits (Maloof 2003, Morgante and Salamini 2003, Paran and Zamir 2003, Borevitz and Chory 2004, Ashikari et al. 2005). The recent development of infrastructures for rice genomics and the completion of the rice genome sequencing project (Sasaki et al. 2005, International Rice Genome Sequencing Project 2005) have greatly facilitated QTL analysis in rice. Thus, we employed QTL analysis to identify the gene(s) involved in rapid internode elongation in deepwater rice.

Since QTL detection is based on natural allelic differences between the selected parental lines; the use of both wild rice species with deepwater characteristics and deepwater cultivars should enable to detect various kinds of QTLs, because wild species exhibit unique geographic and environmental adaptations. Thus, for our QTL analysis, we produced two $\mathrm{F}_{2}$ populations using two rice species display deepwater characteristics: $O$. sativa, a deepwater cultivar, and $O$. rufipogon, a wild rice species. In the present study, we detected and compared multiple QTLs from cultivated $O$. sativa and $O$. rufipogon that were involved in rapid internode elongation in response to water rise. We also produced a NIL carrying a major QTL in a non-deepwater rice background and evaluated the function of this QTL.

\section{Materials and Methods}

\section{Plant materials}

A wild rice species, W0120 (O. rufipogon; perennial type), originally from Cuttack, Orissa, India, which is tolerant to deepwater (Morishima et al. 1962), and a deepwater rice cultivar, C9285 (O. sativa, ssp. indica) originally from Bangladesh, were used as deepwater rice plants in the present study. A wild rice species, W0106 (O. rufipogon; annual type), originally from Phulankara in Bangladesh, and a cultivar, Taichung 65 (T65; O. sativa ssp. japonica) were used as rice plants lacking deepwater character (hereafter referred to as non-deepwater (control) rice plants) and as crossing materials in the present study. Lines C9285, W0120 and W0106 were kindly provided by the National Institute of Genetics in Japan (http://www.shigen.nig.ac.jp/rice/ oryzabase/top/top.jsp). T65 is maintained at Nagoya University.

\section{Development of $F_{2}$ populations and a NIL}

Two sets of two independent $\mathrm{F}_{2}$ populations (i.e., four $\mathrm{F}_{2}$ populations in total) derived from crosses of T65 with C9285 and T65 with W0120 were produced for QTL analysis. To produce a NIL harboring the target QTL region from W0120 in a T65 background, $F_{1}$ plants (T65/W0120) were backcrossed four times by T65. A NIL was selected from the $\mathrm{BC}_{4} \mathrm{~F}_{2}$ generation by marker-assisted selection (MAS) (Yano and Sasaki 1997, Yano 2001).

\section{Phenotypic evaluation}

To evaluate the elongation of the rice plants, we selected three parameters: total internode elongation length (TIL), number of elongated internodes (NEI), and the lowest elongated internode (LEI) position on the main tiller (Fig. 2A). To investigate each trait, the plants were germinated in Petri dishes in water at $30^{\circ} \mathrm{C}$ for $72 \mathrm{~h}$ and transplanted to pots $(10$ $\mathrm{cm}$ in diameter, $12 \mathrm{~cm}$ in height). To determine TIL and NEI, plants were grown in air (i.e., approximately $5 \mathrm{~cm}$ of water); and at the ten-leaf stage, the plants were submerged in water up to $70 \%$ of the plant height in $3,000-\mathrm{L}$ tanks. Thereafter, the water level was increased by $10 \mathrm{~cm}$ every 3 days for 2 weeks, and then the TIL and NEI were determined. Two $\mathrm{F}_{2}$ populations, each consisting of 94 plants derived from crosses of T65 with C9285 and T65 with W0120, were used for QTL analyses of TIL and NEI.

To determine the LEI position, seeds were pregerminated $\left(30^{\circ} \mathrm{C}, 72 \mathrm{~h}\right)$, and transplanted to plug plates (each plug measured $125 \mathrm{~cm}^{3}$ ). At the five-leaf stage, the plants were immersed in a deepwater tank up to about $70 \%$ of the plant height for 1 week. The position of the lowest elongated internode longer than $5 \mathrm{~mm}$ was defined as the LEI position and determined (Inoue 1987).

\section{Construction of linkage maps and QTL analysis}

To construct linkage maps, genomic DNA was extracted from 94 individuals from each $\mathrm{F}_{2}$ population using the TPS method as follows. Rice leaf tips were harvested, and ground using a MULTI-BEADS SHOCKER (Yasui Kikai, Osaka) with TPS buffer (100 mM Tris- $\mathrm{HCl}$ [pH 8.0], $1 \mathrm{M}$ $\mathrm{KCl}$, and $10 \mathrm{mM}$ EDTA). After centrifugation, the supernatant was recovered and an equal volume of isopropyl alcohol was added. The isopropyl alcohol-insoluble material was recovered by centrifugation and the pellet was washed with $75 \%$ ethanol. Thereafter, the pellet was dried and dissolved in TE (10 mM Tris-HCl [pH 8.0] and $1 \mathrm{mM}$ EDTA). The extracted DNA samples were used for genotyping with molecular markers. PCR-based markers, including simplesequence repeat markers (McCouch et al. 2002, Ware et al. 2002, http://www.gramene.org/) and cleaved amplified polymorphic sequence markers (Konieczny and Ausubel 1993), which we selected by comparing the genomic sequence of each parent, were used to construct linkage maps. PCR was performed as described by Chen et al. (1997). The amplified products from each population were separated on $3 \%$ agarose gels in $0.5 \times$ TBE buffer and visualized using 
ethidium bromide. To construct the linkage map, the program MAPMAKER/EXP, version 3.0 (Lander et al. 1987) was used. To identify QTLs for TIL, NEI and LEI, QGene version 3.06 (Nelson 1997) was employed. The presence of QTLs was confirmed declared when the LOD score was 3.0 or higher. One- and two-locus tests were performed to assess QTL interactions.

\section{Results}

\section{Phenotypic evaluation of deepwater rice}

Two types of rice plants were used in the present study: the deepwater rice cultivar C9285 (O. sativa, ssp. indica) and the wild rice species W0120 (O. rufipogon), which exhibit deepwater characteristics. As controls, we used a nondeepwater rice cultivar, T65 (O. sativa, ssp. japonica), and a wild rice species, W0106 (O. rufipogon). When grown in air, none of the lines showed significant internode elongation (Fig. 1). In contrast, growth under deepwater conditions for 2 weeks induced significant internode elongation in the C9285 and W0120 plants, while a slight internode elongation was observed in the T65 or W0106 plants.

To assess the elongation capacity of deepwater rice, Vergara and Mazaredo (1979) proposed the measurement of the total amount of internode elongation. On the other hand, Inoue and Mogami (1980) reported that the position of the lowest elongated internode varied among deepwater rice varieties. Therefore, Inoue (1983) suggested that the position of the lowest elongated internode could be determined to evaluate the deepwater characteristics of rice plants. We adopted these parameters to quantify the deepwater response of the rice plants used. Specifically, we measured the total internode elongation length (TIL) and determined the posi- tion of the lowest elongated internode (LEI; Fig. 2A). Additionally, another parameter, the number of elongated internodes (NEI), was also determined, since extremely long stems in deepwater rice can theoretically result from internode elongation or from an increased number of internodes (Fig. 2A).

To investigate internode elongation in deepwater rice, we determined the TIL, NEI and LEI in air- and deepwatergrown plants. While TIL was not significantly different between the deepwater (i.e., C9285 and W0120) and nondeepwater (i.e., T65 and W0106) plants grown in air, differences were detected between the groups following growth for 2 weeks under deepwater conditions (Fig. 2B). Although internode elongation slightly increased in the non-deepwater plants $(9 \mathrm{~cm}$ in T65 and $12.5 \mathrm{~cm}$ in W0106), the increase among the deepwater plants was much more conspicuous (94.5 cm in C9285 and $77.2 \mathrm{~cm}$ in W0120). A significant difference was also observed between the two groups in terms of the NEI values. Deepwater conditions slightly increased the NEI values in the non-deepwater plants (1.5 in T65 and 2 in W0106), while the NEI values in the deepwater plants were significantly higher (7 in C9285 and 4.5 in W0120; Fig. 2C).

No LEI was detected among the air-grown plants, because no elongation occurred (Fig. 2D). After 1-week of growth under deepwater conditions, the LEI values of the C9285 and W0120 plants were 5.7 and 6.7, respectively, while no LEI was detected in the T65 or W0106 plants.

These results indicated that the C9285 and W0120 plants exhibited typical deepwater characteristics, and that TIL, NEI and LEI were good parameters for assessing the deepwater response.

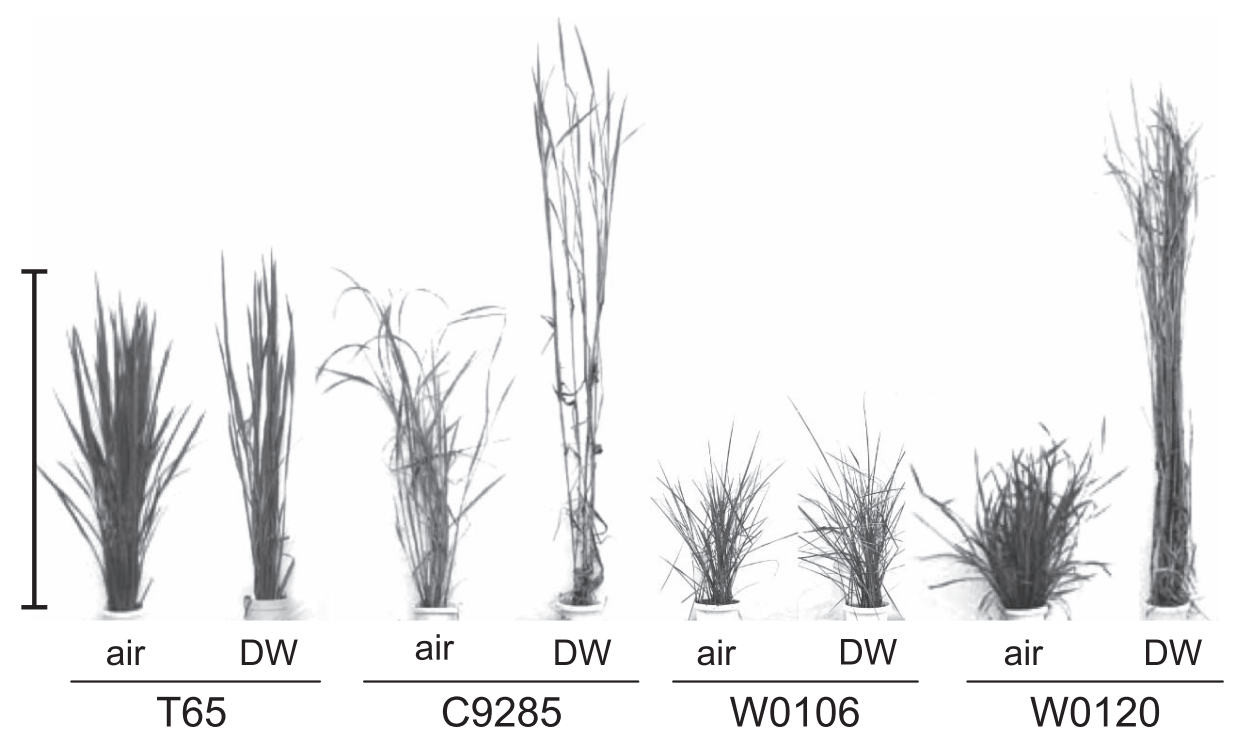

Fig. 1. Response of deepwater rice to deepwater conditions. Two deepwater strains, C9285 (O. sativa, ssp. indica) and W0120 (O. rufipogon), and two non-deepwater strains, T65 (O. sativa, ssp. japonica) and W0106 (O. rufipogon), were grown in air (air) until the ten-leaf stage and then transferred to deepwater conditions (DW) for 2 weeks. Bar, $1 \mathrm{~m}$. 

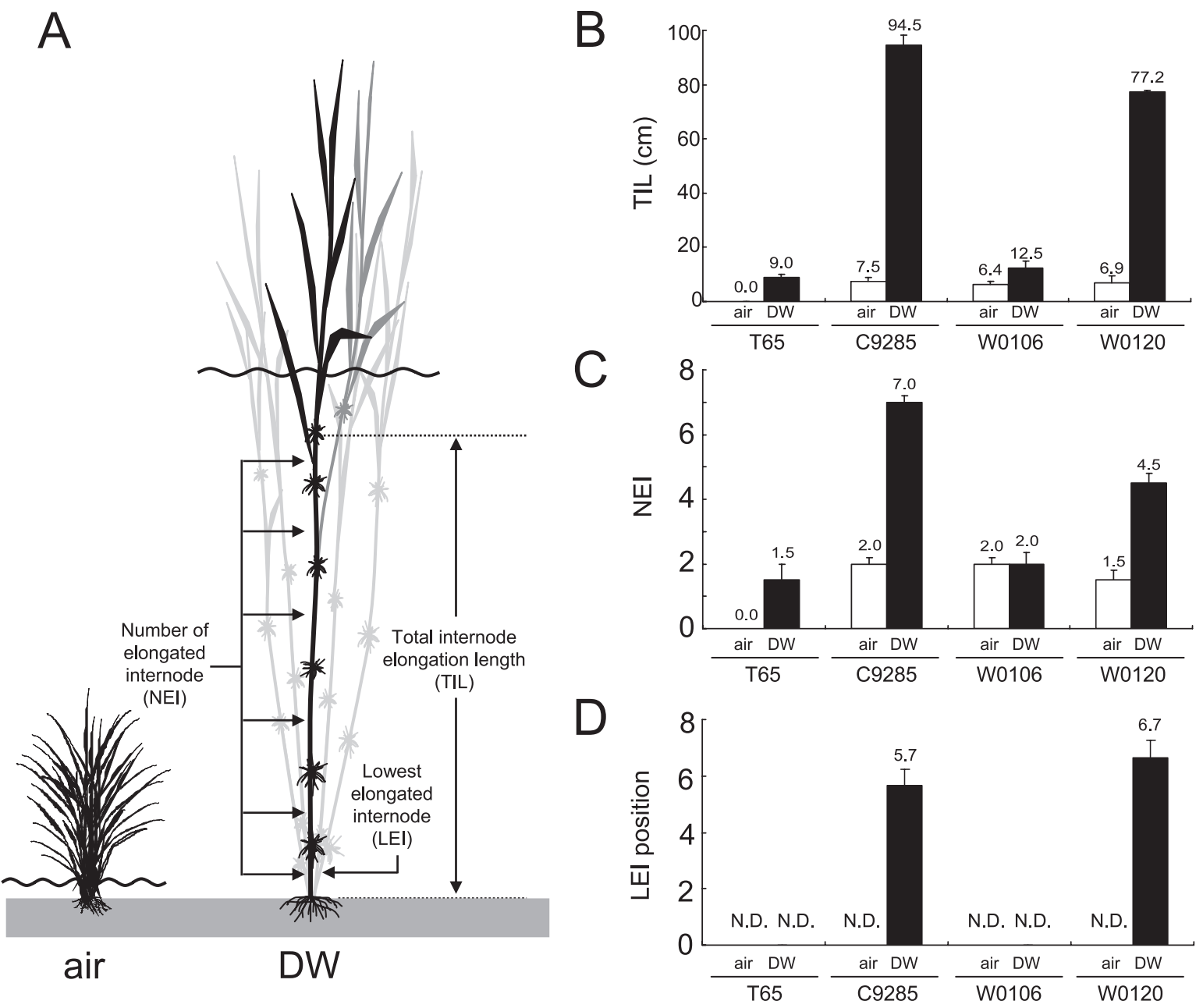

Fig. 2. Quantitative evaluation of internode elongation in deepwater rice. A, Illustration of deepwater rice behavior in air or in deepwater. Total internode elongation length (TIL) corresponds to the length from the base to the highest node. Number of elongated internodes (NEI) corresponds to the total number of elongated internodes. Lowest elongated internode (LEI) position corresponds to the internode position at which internode elongation is initiated. B, Comparison of TILs. C, Comparison of NEIs. D, Comparison of LEIs. Values in B, C and D are means with S.D. $(n=5)$.

\section{Distribution of TIL, NEI and LEI in an $F_{2}$ population}

In QTL analysis, the choice of parental lines is very important, because wide phenotypic variations among the target traits are essential for identifying major QTLs. The use of deepwater rice cultivars and wild rice species as parental lines increases the chance of detecting various types of QTLs, and comparing the QTLs between cultivated deepwater rice and wild species with deepwater characteristics may enable to explain the evolution and domestication of specific attributes. We thus selected a deepwater rice cultivar, $\mathrm{C} 9285$ (O. sativa), and a wild rice species, W0120 (O. rufipogon), as parental lines for a QTL analysis of the deepwater response.

Two $\mathrm{F}_{2}$ populations, each consisting of 188 individuals, were produced from T65/C9285 and T65/W0120 for QTL analysis. The $\mathrm{F}_{2}$ populations were divided into two sets, each with $94 \mathrm{~F}_{2}$ plants from $\mathrm{T} 65 / \mathrm{C} 9285$ and $94 \mathrm{~F}_{2}$ plants from T65/W0120. This procedure was adopted because two of the traits being analyzed, TIL (or NEI) and LEI, could not be ex- amined using the same population due to differences in the way the phenotypes were scored. The first set was used for QTL analysis of TIL and NEI, while the second set was used for QTL analysis of LEI.

The first set of $\mathrm{F}_{2}$ plants was screened for phenotypic variations in TIL and NEI. The plants were partially submerged in water for 2 weeks, and then the TIL and NEI values were determined. The TIL values ranged from 21.4 to $73.7 \mathrm{~cm}$ among the T65/C9285 $\mathrm{F}_{2}$ plants (Fig. 3A-1), and from 12.6 to $72.7 \mathrm{~cm}$ among the $\mathrm{T} 65 / \mathrm{W} 0120 \mathrm{~F}_{2}$ plants (Fig. 3A-2). In comparison, the NEI values ranged from 2 to 8 among the T65/C9285 $\mathrm{F}_{2}$ plants (Fig. 3B-1), and from 1 to 5 among the T65/W0120 $\mathrm{F}_{2}$ plants (Fig. 3B-2).

The second set of $F_{2}$ plants was kept under deepwater conditions for 1 week, and then the LEI positions were determined. The LEI values ranged from 6 to 9 among the T65/ C9285 $\mathrm{F}_{2}$ plants (Fig. 3C-1), and from 7 to 10 among the T65/W0120 $\mathrm{F}_{2}$ plants (Fig. 3C-2). The LEI position in some lines from each $F_{2}$ population could not be determined 
A (1)

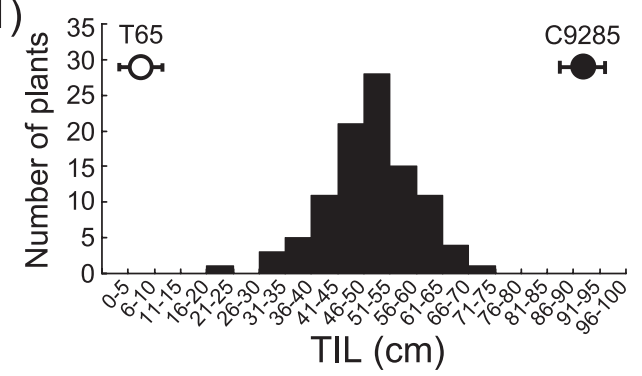

B (1)

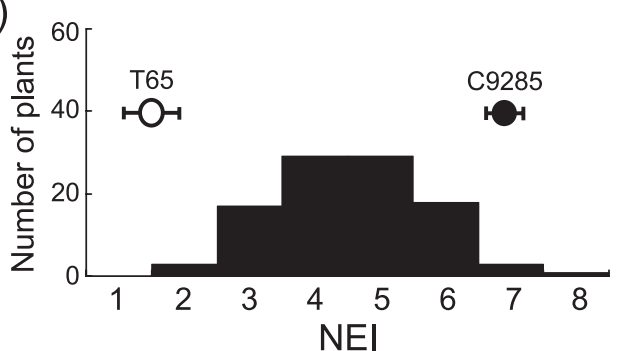

C (1)

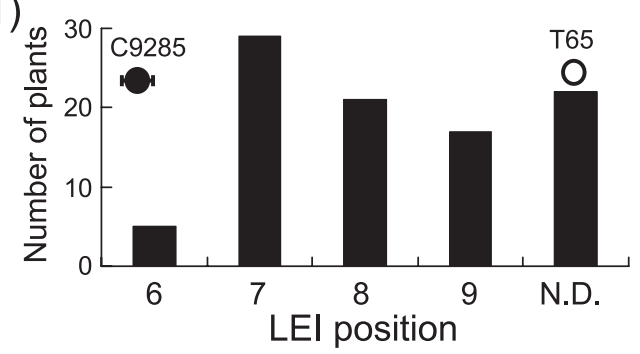

(2)

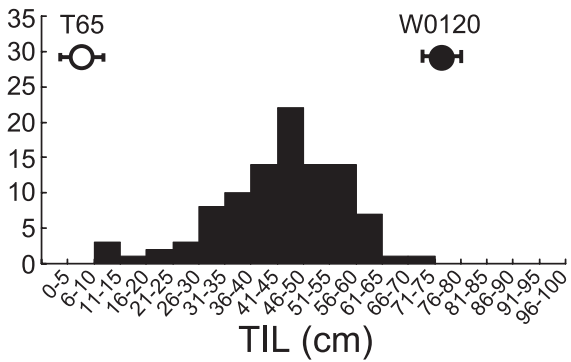

(2)

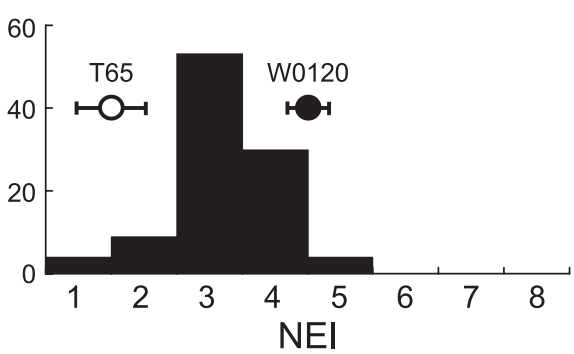

(2)

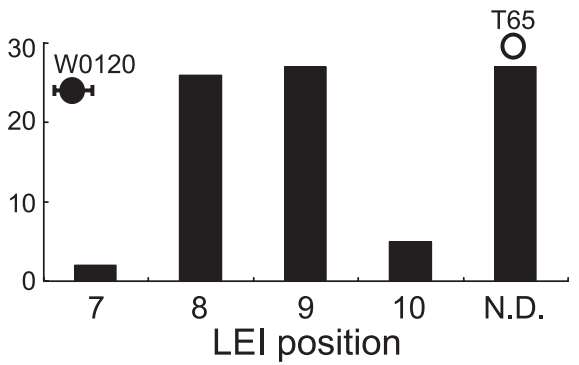

Fig. 3. Distribution frequencies of TIL, NEI and LEI for $94 \mathrm{~F}_{2}$ individuals. A, Distribution of TIL among $\mathrm{F}_{2}$ plants from T65/C9285 (1) and T65/W0120 (2). B, Distribution of NEI among $F_{2}$ plants from T65/C9285 (1) and T65/W0120 (2). C, Distribution of LEI among $\mathrm{F}_{2}$ plants from T65/C9285 (1) and T65/W0120 (2). O, T65; Deepwater rice.

(N.D.), due to the absence of no elongation (Fig. 3C).

Even though the distribution ranges for TIL in the T65/ C9285 and T65/W120 $\mathrm{F}_{2}$ populations became smaller than those of the parents, a continuous distribution was recorded, and a continuous distribution of each trait (i.e., NEI and LEI) was also observed among our $F_{2}$ populations. The lack of a $3: 1$ or $1: 2: 1$ distribution indicated that these traits were controlled by QTLs and not by a single locus.

\section{Construction of linkage maps for QTL analysis}

Linkage maps, which are an essential component of QTL analyses, were constructed from each set of $F_{2}$ plants (first set, Fig. 4A and B; second set, data not shown). Ninetytwo molecular markers spaced at an average interval of 19.1 cM were used to construct a linkage map for the T65/C9285 population (Fig. 4A); and 106 markers spaced at an average interval of $17.0 \mathrm{cM}$ were used for the T65/W0120 population (Fig. 4B). Since the molecular markers were equally spaced and that they covered the entire genome, QTLs with large effects located anywhere within the rice genome were likely to be revealed by our analysis.

\section{QTL analysis of TIL, NEI and LEI}

Using our $\mathrm{F}_{2}$ phenotypic data and linkage maps, QTL analyses were performed for TIL, NEI and LEI. Two QTLs,

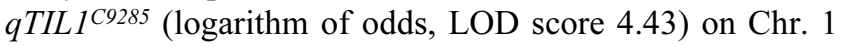
and $q$ TIL $12^{C 9285}$ (LOD score 6.19) on Chr. 12, were detected in the $\mathrm{T} 65 / \mathrm{C} 9285 \mathrm{~F}_{2}$ plants, and the $\mathrm{C} 9285$ allele enhanced TIL (Table 1 and Fig. 4A). Three QTLs, qTIL1 $1^{\text {T65 }}$ (LOD score 3.86) on Chr. 1, qTIL8 ${ }^{T 65}$ (LOD score 3.36) on Chr. 8 and $q T I L 12^{W 0120}$ (LOD score 5.89) on Chr. 12, were detected in the T65/W0120 $\mathrm{F}_{2}$ plants (Table 1 and Fig. 4B). The W0120 allele of $q T I L 12^{W 0120}$ and the T65 alleles of $q T I L 1^{T 65}$ and $q T I L 8^{T 65}$ enhanced TIL. These results, taken from two independent populations, demonstrated that the strongest QTL for TIL in deepwater rice was located on the long arm of Chr. 12.

We next searched for QTLs for NEI. A single QTL, which was detected in the T65/C9285 (qNEI12C9285 (LOD score 6.25)) and T65/W0120 (qNEI12 $2^{W 0120}$ (LOD score 4.47)) $\mathrm{F}_{2}$ populations, respectively (Table 1 and Fig. 4A, B), showed similar positions on the long arm of Chr. 12 in deepwater rice.

For LEI, two QTLs, $q L E I 3^{C 9285}$ (LOD score 3.14) on Chr. 3 and $q L E I 12^{C 9285}$ (LOD score 7.75) on Chr. 12, were 


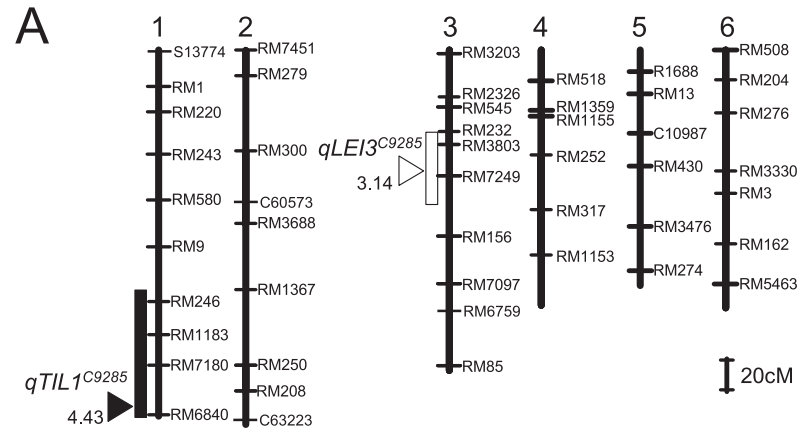

B
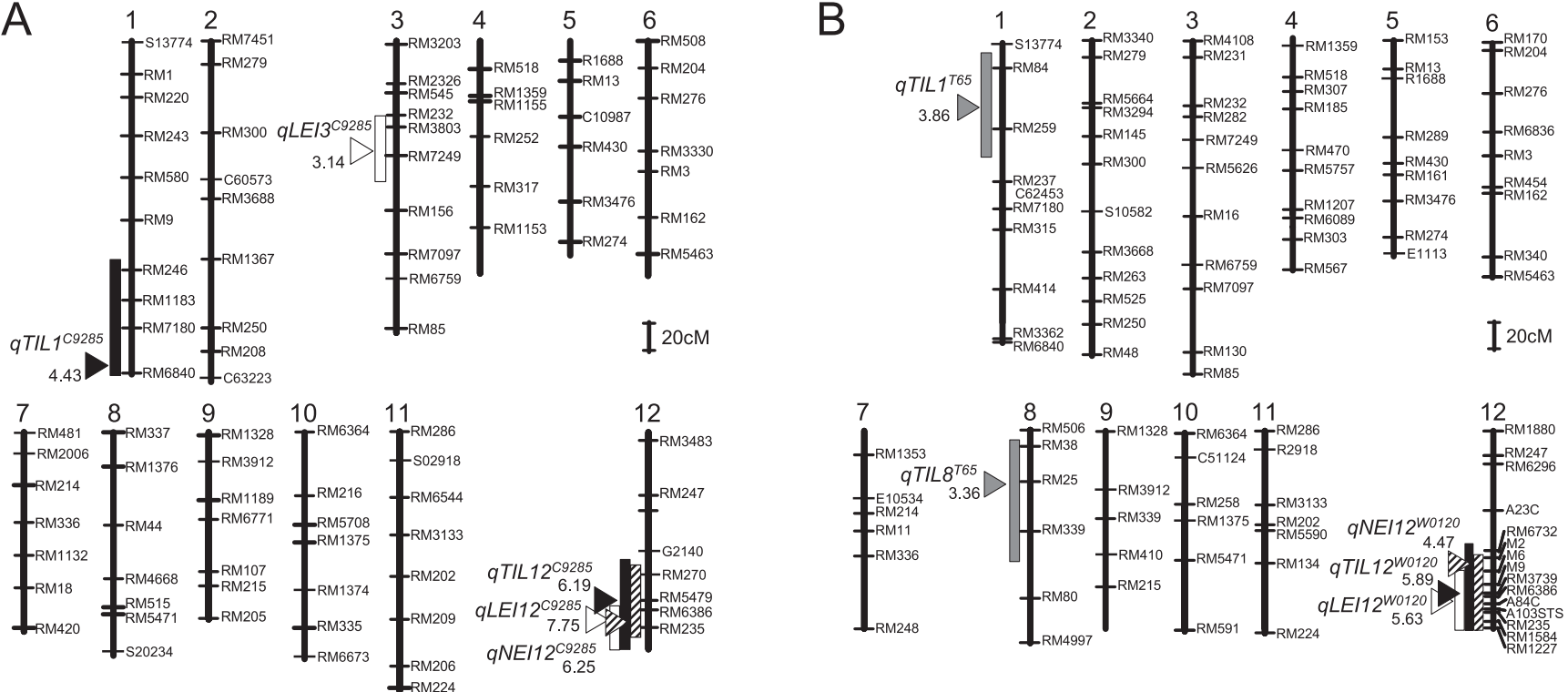

Fig. 4. QTLs for TIL, NEI and LEI. A, Location of QTLs for TIL, NEI and LEI on the linkage map from T65/C9285. B, Location of QTLs for TIL, NEI and LEI on the linkage map from T65/W0120. The region of the QTL for TIL enhanced by the deepwater allele is illustrated by a black box. The region of the QTL for TIL enhanced by the T65 allele is illustrated by a dotted box. The region of the QTL for NEI enhanced by the deepwater allele is illustrated by a hatched box. The region of the QTL for LEI enhanced by the deepwater allele is illustrated by a white box. Arrowheads indicate the QTL peaks with the LOD scores.

detected in the T65/C9285 population, and the C9285 alleles enhanced LEI (Table 1 and Fig. 4A, B). A single QTL, $q L E I 12^{W 0120}$, on Chr. 12 (LOD score 5.63), was detected in the T65/W0120 population, and the W0120 allele enhanced LEI. As previously indicated, although our analysis enabled to detect several QTLs, one- and two-locus tests failed to identify any interaction between them (data not shown).

\section{Production of NILs and QTL evaluation}

To evaluate the effect of individual QTLs, it is necessary to produce NILs for each one in a common genomic background, since the NIL carrying the target QTL can eliminate the effects of the other QTLs (Paterson et al. 1988). All the major QTLs identified in our analysis were detected in the same region on the long arm of Chr. 12 (Fig. 4A, B). To demonstrate the existence of the QTLs and to evaluate their effects, we produced a NIL carrying the QTLs from W0120 in a non-deepwater background by repeated backcrossing to T65. NIL-12 ${ }^{\text {W0120 }}$, which carried a small chromosome fragment (between molecular markers, M2 and RM235: 28.4 $\mathrm{cM}$ ) corresponding to the long arm of Chr. 12, specifically introgressed from W0120, was screened as a NIL by MAS in the $\mathrm{BC}_{4} \mathrm{~F}_{2}$ population (Fig. 5A; Yano 2001, Ashikari and Matsuoka 2006).

Immersion for two weeks induced a dramatic internode elongation in the NIL-12 ${ }^{\text {W0120 }}$ plants (Fig. 5B). The TIL and NEI values in the NIL- $12^{\text {w0120 }}$ plants $(62.6 \mathrm{~cm}$ and 4.0 , respectively) were slightly lower than those observed for

Table 1. Putative QTLs for TIL, NEI and LEI traits

\begin{tabular}{|c|c|c|c|c|c|c|}
\hline Trait & Population & QTL name & NML & Source & PVE & LOD score \\
\hline \multirow[t]{5}{*}{ TIL } & C9285 & $q T I L 1$ & RM6840 & C9285 & $22 \%$ & 4.43 \\
\hline & & qTIL12 & RM235 & C9285 & $27 \%$ & 6.19 \\
\hline & W0120 & qTIL1 & RM259 & T65 & $20 \%$ & 3.86 \\
\hline & & qTIL8 & RM25 & $\mathrm{T} 65$ & $17 \%$ & 3.36 \\
\hline & & qTIL12 & RM6386 & W0120 & $36 \%$ & 5.89 \\
\hline \multirow[t]{2}{*}{ NEI } & C9285 & $q N E I 12$ & RM235 & C9285 & $27 \%$ & 6.25 \\
\hline & W0120 & $q N E I 12$ & M2 & W0120 & $27 \%$ & 4.47 \\
\hline \multirow[t]{3}{*}{ LEI } & C9285 & $q L E I 3$ & RM7249 & C9285 & $14 \%$ & 3.14 \\
\hline & & qLEI12 & RM235 & C9285 & $36 \%$ & 7.75 \\
\hline & W0120 & $q L E I 12$ & RM6386 & W0120 & $26 \%$ & 5.63 \\
\hline
\end{tabular}

QTL names are designated with the abbreviation of the trait name and chromosome number. NML, nearest marker locus of putative QTLs; source, phenotypic direction to which an allele enhances a trait respectively; PVE, phenotypic variance explained by each QTL; LOD, logarithm of odds score. 


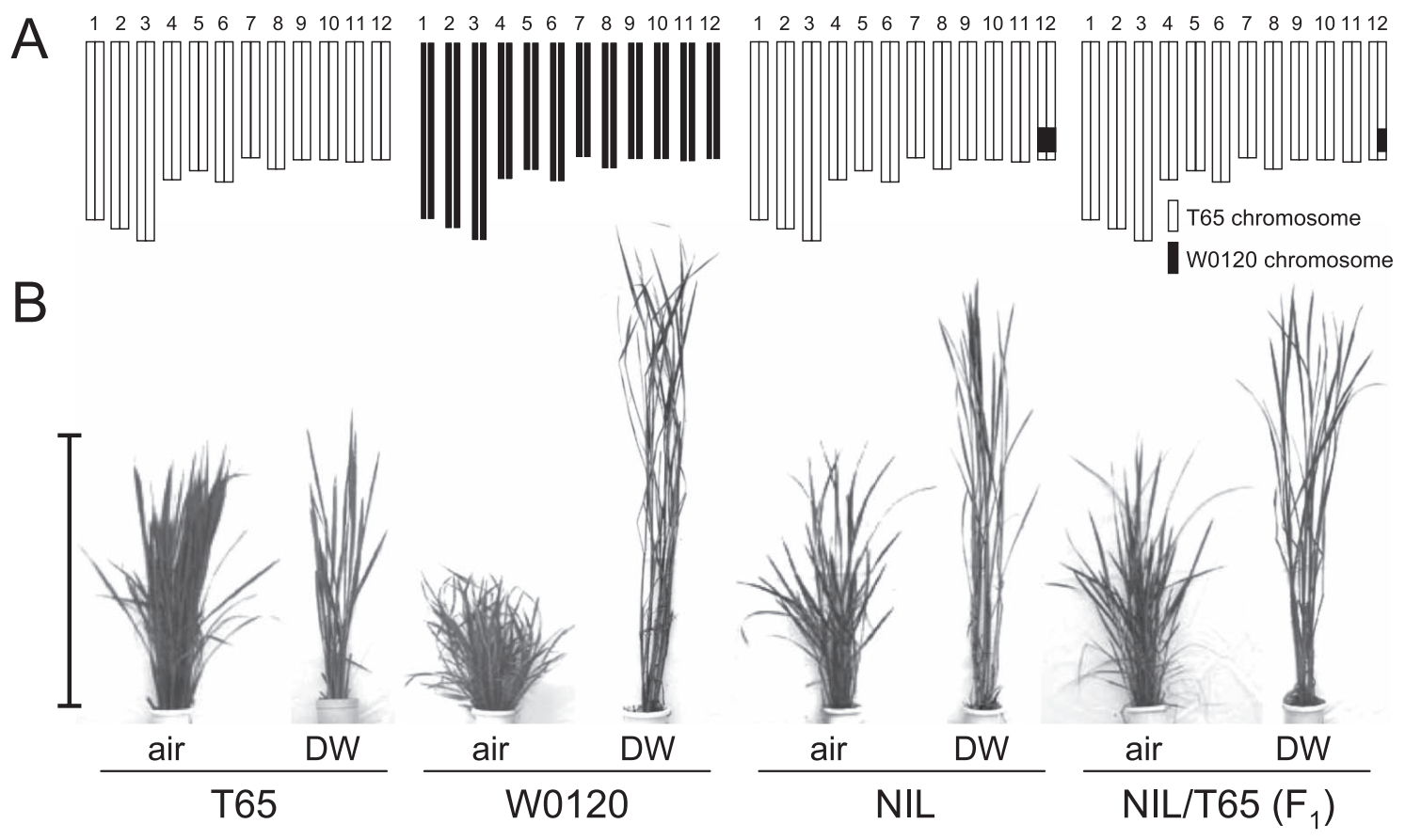

Fig. 5. Response of the NIL- $12^{\mathrm{W} 0120}$ plants to water rise. A, Graphical genotype. From left to right: T65, W0120, NIL and $\mathrm{F}_{1}$ (NIL/T65). NIL-12 ${ }^{\text {W0120 }}$ is abbreviated as NIL. Open bars indicate T65 chromosomes. Closed bars designate W0120 chromosomes. B, Internode elongation in water. Bar, $1 \mathrm{~m}$. air, air condition; DW, deepwater condition.

W0120 ( $76.3 \mathrm{~cm}$ and 4.7, respectively), but they were significantly higher than those of the recurrent parent $\mathrm{T} 65(8.5 \mathrm{~cm}$ and 1.3, respectively; Fig. 6A, B). The LEI position in the NIL-12 ${ }^{\text {W0120 }}$ plants was close to that of the W0120 plants (data not shown). These results indicated that the introgressed chromosome fragment from W0120 included the QTLs for TIL, NEI and LEI, and that these QTLs exerted significant effects on each trait. Additionally, the $F_{1}$ plants crossed with NIL-12 ${ }^{\mathrm{W} 0120}$ and T65 responded to water with an increased TIL value $(60.4 \mathrm{~cm})$ and NEI value $(3.7$; Fig. 5 , Fig. 6), and the LEI position was similar to that in the W0120 plants (data not shown). These results indicated that all of the QTLs were inherited in a dominant manner.

\section{Discussion}

\section{Internode elongation in C9285 and W0120}

We used three parameters, TIL, NEI and LEI, to quantify the internode elongation of deepwater rice. Deepwater conditions which dramatically changed the value of TIL, NEI and LEI position in deepwater rice (C9285 and W0120) did not affect those of non-deepwater rice (T65 and W0106; Fig. 2B, C and D). Each of the three parameters was significantly different between the deepwater and non-deepwater rice plants. Our results clearly indicated that TIL, NEI and LEI are suitable parameters for evaluating deepwater responses and for QTL analyses.

\section{Detection of QTLs for rapid internode elongation}

QTL analysis for TIL, NEI and LEI using two different
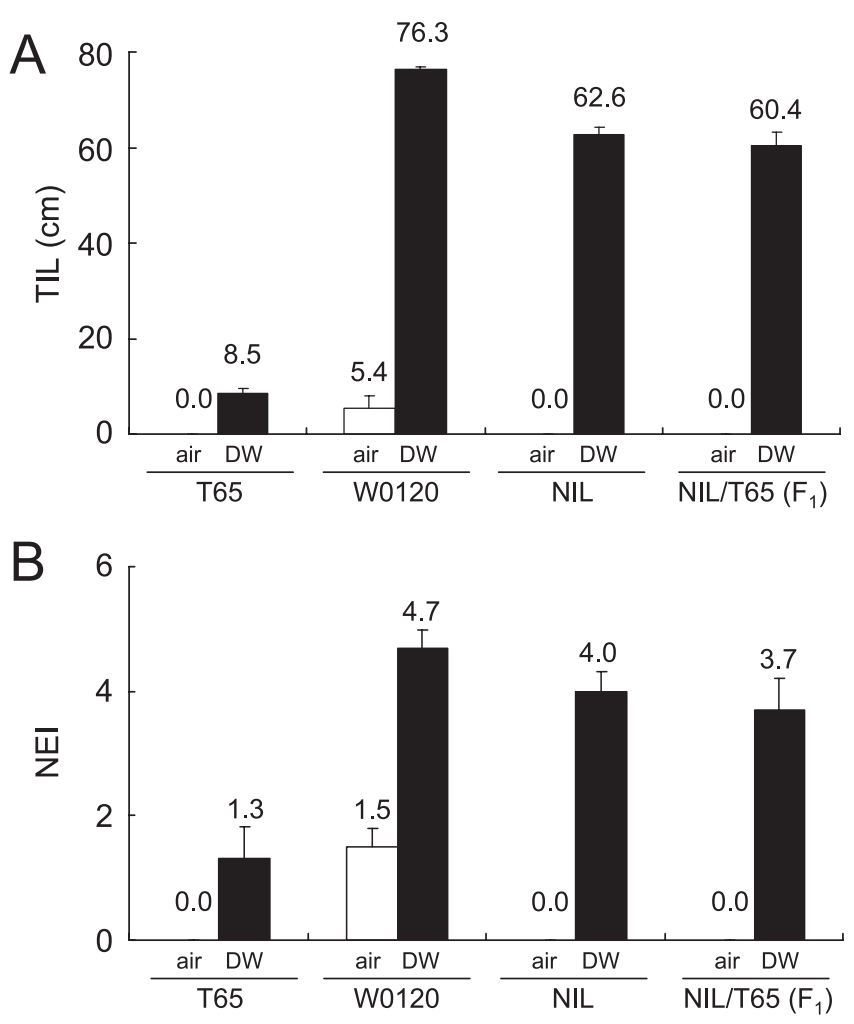

Fig. 6. Quantitative evaluation of the response of the NIL-12 ${ }^{\text {W0120 }}$ plants to water rise. A, Quantitative internode elongation, total internode elongation length (TIL). B, Quantitative internode elongation, number of elongated internodes (NEI). Values in A and $B$ are means with S.D. $(n=5)$. air, air condition; DW, deepwater condition. NIL, NIL-12 ${ }^{\text {W0120. }}$. 
$\mathrm{F}_{2}$ populations (from T65/C9285 and T65/W0120) enabled to detect a total of ten QTLs (LOD score >3.0) (Fig.4). Among them, the strongest QTLs for each trait in both populations were enhanced by the deepwater allele, and they were located in a common region on the long arm of Chr. 12 . Nemoto et al. (2004) also detected a QTL for LEI characters on Chr. 12. Together, these results suggested that the most important QTLs for internode elongation in deepwater rice were located in this region. Additionally, our QTLs were detected in both a deepwater rice cultivar (C9285) and in a wild rice species (W0120). These results suggested that the QTLs on Chr. 12 that mediate internode elongation in response to water rise were shared by a deepwater cultivar of O. sativa and the wild rice species O. rufipogon (W0120).

As shown in Fig. 2 (B and C), a slight increase in the TIL and NEI values was observed in deepwater rice plants (7.5 $\mathrm{cm}$ and 2.0 for C9285 and $6.9 \mathrm{~cm}$ and 1.5 for W0120), even in air. Such a slight increase may have been caused by the shallow water conditions $(<5 \mathrm{~cm}$, see Materials and Methods), which are normal for rice. The extreme sensitivity of deepwater rice to water rise may lead to the early initiation of internode elongation, even in shallow water. On the other hand, T65, a non-deepwater rice variety, also showed a muted response to deepwater as evidenced, by the slight increase of both TIL $(9 \mathrm{~cm})$ and NEI values (1.5; Fig. 2B, C), although the response was much weaker than that of the deepwater plants. Among the ten detected QTLs, two minor QTLs were enhanced by the T65 alleles. Slight increases in the TIL and NEI values may depend on these QTLs, which are intrinsic to $\mathrm{T} 65$.

\section{A single major QTL regulates deepwater characteristics}

To date, in most studies of deepwater rice, the physiological response of plants grown in air had been compared with that of plants grown under deepwater conditions. However, since the stress caused by immersion can affect the expression of many genes in addition to the physiological conditions of the plants, it may be difficult to identify the specific gene or physiological event affected. NILs are a valuable tool for addressing this problem. A NIL carrying the target QTL in a non-deepwater rice background can be used to investigate exactly which gene and/or physiological event is affected by comparing the response of the NIL with that of non-deepwater rice under deepwater conditions. To identify and characterize the QTLs involved in the deepwater response in rice, we produced NIL-12 ${ }^{\mathrm{W} 0120}$, which carries the strongest QTL region from W0120 in a non-deepwater rice (T65) background, by repeated backcrossing using MAS. The TIL and NEI values of the NIL-12 ${ }^{\text {W0120 }}$ plants $(62.6 \mathrm{~cm}$ and 4.0 , respectively) were similar to those of the W0120 plants $(76.3 \mathrm{~cm}$ and 4.7 , respectively), indicating that the major QTL exerted a significant effect on internode elongation. The remaining difference in the TIL and NEI values between the NIL-12 ${ }^{\mathrm{W} 0120}$ and W0120 plants may be associated with other minor QTLs on Chrs. 1 and 3. Oneand two-locus tests did not reveal any interaction between the major QTL on Chr. 12 and the other QTLs, such as those on Chrs. 1 and 3. Since the $F_{2}$ populations used in the present study may have been too small to detect interactions between the QTLs, NILs should be produced for each QTL to allow pyramiding in order to investigate the relationship among QTLs (Ashikari et al. 2005, Ashikari and Matsuoka 2006). We are currently producing NILs and lines with QTL pyramiding for all the combinations of the QTLs detected in the present study. A comparison of each NIL and line with QTL pyramiding may uncover interactions between the QTLs in deepwater rice.

To understand the molecular mechanism(s) of rapid internode elongation in deepwater rice, it is necessary to clone the QTL. The NIL-12 ${ }^{\mathrm{W} 0120}$ heterozygote showed the same TIL, NEI and LEI values as those of the NIL (Fig. 6), which suggests that the major QTL is a dominant gene. In several reports, genes that induce rapid internode elongation in deepwater rice have been identified. For example, deepwater characteristics have been reported to be controlled by duplicate genes (ef1 and ef2; Ramiah and Ramaswami 1940), by a partially dominant gene (Hamamura and Kupkanchankul 1979), by a single dominant gene (Tripathi and Balakrishna Rao 1985), by an incompletely dominant gene (Suge 1987) and by a single recessive gene ( $d w 3$; Eiguchi et al. 1993). Despite these reports, however, the exact genes that regulate internode elongation under deepwater conditions have not yet been identified. In the present study, we detected QTLs for deepwater characteristics. Although some QTLs were weak, the production and evaluation of a NIL with mapping of the QTLs demonstrated that a major QTL was sufficient to regulate rapid internode elongation in deepwater rice. At present, we cannot determine whether the most effective QTLs detected on the long arm of Chr. 12 represented the same gene or multiple genes. However, the fact that the QTL analysis detected these QTLs to the same chromosomal region, that all behaved in a dominant manner and that they were detected in both a deepwater cultivar and in a wild species suggested that all of these traits may be regulated by a single dominant gene. Identification of the QTL by positional cloning is currently underway.

\section{The major QTL switches on the machinery for rice internode elongation}

By comparing how the NIL-12 ${ }^{\mathrm{w} 0120}$ and T65 plants responded to water rise, we were able to predict the exact target of the major QTL and to speculate how it functions. The NIL-12 20120 plants exhibited rapid internode elongation, with TIL and NEI values being close to those of the original deepwater rice (Fig. 6). The introduction of the QTL from W0120 induced deepwater rice characteristics in a nondeepwater rice variety (T65), indicating that the QTL was sufficient to confer the deepwater response. Rapid internode elongation in deepwater rice may be regulated by several successive steps, from the perception of flooding to stem elongation, and many genes may take part in the water stress response. However, our results indicated that rapid internode 
elongation in deepwater rice compared with that in nondeepwater rice was controlled by the QTL. The introduction of the QTL into a non-deepwater rice background resulted in rapid internode elongation similar to that observed in deepwater rice. This indicates that the major QTL encodes a critical gene that is lacking in non-deepwater rice, and that the other genes required for rapid internode elongation are shared between non-deepwater and deepwater rice. In other words, deepwater and non-deepwater rice share the same machinery for stem elongation, but deepwater rice is specifically adapted for flood survival due to the presence of a single gene that switches on the machinery in response to water rise. The QTL identified in the present study may be the master trigger for rapid internode elongation during flooding in deepwater rice.

\section{Domestication of the major QTL}

It is possible that only some strains of $O$. rufipogon are able to elongate internodes in response to deepwater conditions. In fact, one of them, W0106, did not respond to water rise by internode elongation. Numerous accessions of $O$. rufipogon that display a broad phenotypic diversity have been collected from a range of monsoonal areas across Asia (Vaughan 1994). There are different groups of O. rufipogon, and some exhibit the ability to elongate their internodes in response to water rise unlike others. It is likely that the ancestor of $O$. rufipogon acquired this function to survive during flooding and then became adapted to flooded habitats. Some groups of $O$. rufipogon may have subsequently lost this function following their introduction into non-flooded areas.

Most non-deepwater rice cultivars may have lost the ability to regulate their internode elongation in response to water rise because the trait is unnecessary, due to the advent of crop management and irrigation. It has long been considered that Asian cultivated rice (O. sativa) evolved from a wild species (O. rufipogon; Cheng et al. 2002, 2003, Vaughan et al. 2003). Our results showing that the same chromosomal region in cultivated $(O$. sativa, $\mathrm{C} 9285)$ and wild (O. rufipogon, W0120) rice is essential for internode elongation and that the major QTL is dominant, led us to speculate that internode elongation in response to water rise originated in O. rufipogon and was transmitted to cultivated O. sativa. On the other hand, in cultivars such as C9285 that are frequently planted in flooded areas this function has been maintained.

To understand how the ability of the rice plants to elongate internodes in response to water rise was acquired and transmitted, cloning of the QTL and sequence comparisons among wild rice species and deepwater cultivars of $O$. sativa should be performed. Additionally, continuation of physiological studies on our NIL and cloning of the QTL may enable to elucidate the mechanism(s) underlying the deepwater response, and may also contribute to understanding the mechanism(s) used by other aquatic or semi-aquatic plants.

\section{Acknowledgments}

We thank Professor Nori Kurata and Dr. Mitsugu Eiguchi for providing W0120 and C9285 seeds. We also thank Professor Atsushi Yoshimura and Hikaru Sato for providing the rice mutagenized line. This work was supported by a grant from the Ministry of Agriculture, Forestry and Fisheries of Japan (MAFF) (Green Technology Project QT-2003) and Research Fellowships from the Japan Society for the Promotion of Science for Young Scientists.

\section{Literature Cited}

Ashikari, M., H. Sakakibara, S. Lin, T. Yamamoto, T. Takashi, A. Nishimura, E.R.Angeles, Q.Qian, H.Kitano and M.Matsuoka (2005) Cytokinin oxidase regulates rice grain production. Science 309: 741-745.

Ashikari, M. and M.Matsuoka (2006) Identification, isolation and pyramiding of quantitative trait loci for rice breeding. Trends. Plant Sci. 11: 344-350.

Borevitz,J.O. and J.Chory (2004) Genomics tools for QTL analysis and gene discovery. Curr. Opin. Plant Biol. 7: 132-136.

Catling, D. (1992) Rice in deep water. International Rice Research Institute, Manila.

Chen, X., S.Temnykh, Y.Xu, Y.G.Cho and S.R.McCouch (1997) Development of a microsatellite framework map providing genome-wide coverage in rice (Oryza sativa L.). Theor. Appl. Genet. 95: 553-567.

Cheng,C., S.Tsuchimoto, Y.Fukuta, H.Ohtsubo and E.Ohtsubo (2002) Evolutionary relationships among rice species with AA genome based on SINE insertion analysis. Genes Genet. Syst. 77: 323-334.

Cheng, C., R.Motohashi, S.Tsuchimoto, Y.Fukuta, H.Ohtsubo and E.Ohtsubo (2003) Polyphyletic origin of cultivated rice: based on the interspersion pattern of SINEs. Mol. Biol. Evol. 20: 6775.

Cho,H.T. and H.Kende (1997) Expansins in deepwater rice internodes. Plant Physiol. 113: 1137-1143.

Eiguchi,M., H.Y.Hirano, Y.Sano and H.Morishima (1993) Effect of water depth on internode elongation and floral induction in a deepwater-tolerant rice line carrying the dw3 gene. Jpn. J. Breed. 43: 135-139.

Hamamura,K. (1993) Floating rice. In "Science of the Rice Plant, Vol. 1" Matsuo, T. and H.Hoshikawa (eds.), Food and Agriculture Policy Research Center, Tokyo. p. 66-70.

Hamamura, K. and T.Kupkanchankul (1979) Inheritance of floating ability of rice. Jpn. J. Breed. 29: 211-216.

Inoue, J. (1983) Relation between elongation ability and internode elongation of floating rice under rising water conditions. Jpn. J. Trop. Agr. 27: 181-186.

Inoue, J. (1987) On floating rice and its ecological traits in Southeast Asia. Southeast Asia Studies 25: 51-61 (in Japanese).

Inoue, J. and Y.Mogami (1980) On the position of the lowest internode of floating rices originated in different countries. Jpn. J. Trop. Agr. 24: 13-17.

International Rice Genome Sequencing Project (2005) The map-based sequence of the rice genome. Nature 436: 793-800.

Kende,H., E.van derKnaap and H.T.Cho (1998) Deepwater rice: A model plant to study stem elongation. Plant Physiol. 118: 1105-1110. 
Konieczny, A. and F.M.Ausubel (1993) A procedure for mapping Arabidopsis mutations using co-dominant ecotype-specific PCR-based markers. Plant J. 4: 403-410.

Lander,E.S., P.Green, J.Abrahamson, A. Barlow, M.J.Daly, S.E. Lincoln and L.Newburg (1987) MAPMAKER: an interactive computer package for constructing primary genetic linkage maps of experimental and natural populations. Genomics 1: 174-181.

Lee, Y. and H.Kende (2001) Expression of $\beta$-expansins is correlated with internodal elongation in deepwater rice. Plant Physiol. 127: 645-654.

Lee, Y. and H.Kende (2002) Expression of $\alpha$-expansin and expansinlike genes in deepwater rice. Plant Physiol. 130: 1396-1405.

Maloof,J.N. (2003) QTL for plant growth and morphology. Curr. Opin. Plant Biol. 6: 85-90.

McCouch,S.R., L.Teytelman, Y.Xu, K.B.Lobos, K.Clare, M.Walton, B.Fu, R.Maghirang, Z.Li, Y.Xing, Q.Zhang, I.Kono, M.Yano, R.Fjellstrom, G.DeClerck, D.Schneider, S.Cartinhour, D.Ware and L.Stein (2002) Development and mapping of 2240 new SSR markers for rice (Oryza sativa L.). DNA Res. 9: 199-207.

Métraux,J.P. and H.Kende (1983) The role of ethylene in the growth response of submerged deepwater rice. Plant Physiol. 72: 441446.

Morgante,M. and F. Salamini (2003) From plant genomics to breeding practice. Curr. Opin. Biotechnol. 14: 214-219.

Morishima,H., K.Hinata and H.I.Oka (1962) Floating ability and drought resistance in wild and cultivated species of rice. Indian J. Genet. 22: 1-11.

Nelson,J.C. (1997) QGENE: software for marker-based genomic analysis and breeding. Mol. Breed. 3: 239-245.

Nemoto,K., Y.Ukai, D.Q.Tang, Y.Kasai and M.Morita (2004) Inheritance of early elongation ability in floating rice revealed by diallel and QTL analyses. Theor. Appl. Genet. 109: 42-47.

Paran,I. and D.Zamir (2003) Quantitative traits in plants: beyond the QTL. Trends. Genet. 19: 303-306.

Paterson,A.H., E.S.Lander, J.D.Hewitt, S.Peterson, S.E.Lincoln and S.D. Tanksley (1988) Resolution of quantitative traits into Mendelian factors by using a complete linkage map of restriction fragment length polymorphisms. Nature 335: 721-726.

Ramiah,K. and K.Ramaswami (1940) Floating habit in rice. Indian J. Agric. Sci. 11: 1-8.

Raskin,I. and H.Kende (1984) Regulation of growth in stem sections of deepwater rice. Planta 160: 66-72.

Sasaki,T., T.Matsumoto, B.A.Antonio and Y.Nagamura (2005) From mapping to sequencing, post-sequencing and beyond. Plant Cell Physiol. 46: 3-13.

Suge,H. (1987) Physiological genetics of internode elongation under submergence in floating rice. Jpn. J. Genet. 62: 69-80.
Tripathi, R.S. and M.J.Balakrishna Rao (1985) Inheritance studies of characters associated with floating habit and their linkage relationship in rice. Euphytica 34: 875-881.

VanderKnaap,E., S.Jagoueix and H.Kende (1997) Expression of an ortholog of replication protein A1 (RPA1) is induced by gibberellin in deepwater rice. Proc. Natl. Acad. Sci. USA 94: 9979-9983.

Van der Knaap,E., W.Y.Song, D.L.Ruan, M.Sauter, P.C.Ronald and H.Kende (1999) Expression of a gibberellin-induced leucinerich repeat receptor-like protein kinase in deepwater rice and its interaction with kinase-associated protein phosphatase. Plant Physiol. 120: 559-570.

VanderKnaap,E., J.H.Kim and H.Kende (2000) A novel gibberellininduced gene from rice and its potential regulatory role in stem growth. Plant Physiol. 122: 695-704.

Vaughan,D.A. (1994) The wild relatives of rice: a genetic resources handbook. International Rice Research Institute, Manila.

Vaughan,D.A., H.Morishima and K.Kadowaki (2003) Diversity in the Oryza genus. Curr. Opin. Plant Bio. 16: 139-146.

Vergara,B.S., B.Jackson and S.K.De Datta (1976) Deepwater rice and its response to deepwater stress. Climate and rice. International Rice Research Institute, Manila.

Vergara,B.S. and A.Mazaredo (1979) Using the new standard evaluation system to measuring elongation ability. Proc 1978 Intl deepwater rice workshop, International Rice Research Institute, Manila, p. 139-142.

Vriezen, W.H., R.Hulzink, C.Mariani and L.A.C.J.Voesenek (1999) 1-aminocyclopropane-1-carboxylate oxidase activity limits ethylene biosynthesis in Rumex palustris during submergence. Plant Physiol. 121: 189-196.

Ware,D.H., P.Jaiswal, J.Ni, I.V.Yap, X.Pan, K.Y.Clark, L.Teytelman, S.C.Schmidt, W.Zhao, K.Chang, S.Cartinhour, L.D.Stein and S.R.McCouch (2002) Gramene, a Tool for Grass Genomics. Plant Physiol. 130: 1606-1613.

Yano,M. (2001) Genetic and molecular dissection of naturally occurring variation. Curr. Opin. Plant. Biol. 4: 130-135.

Yano, M. and T.Sasaki (1997) Genetic and molecular dissection of quantitative traits in rice. Plant Mol. Biol. 35: 145-153.

Zarembinski,T.I. and A.Theologis (1997) Expression characteristics of $O S-A C S 1$ and $O S-A C S 2$, two members of the 1-aminocyclopropane-1-carboxylate synthase gene family in rice (Oryza sativa L. cv. Habiganj Aman II) during partial submergence. Plant Mol. Biol. 33: 71-77.

Zhou,Z., W.Vriezen, W.V.Caeneghem, M.V.Montagu and D.Vander Straeten (2001) Rapid induction of a novel ACC synthase gene in deepwater rice seedlings upon complete submergence. Euphytica 121: 137-143. 